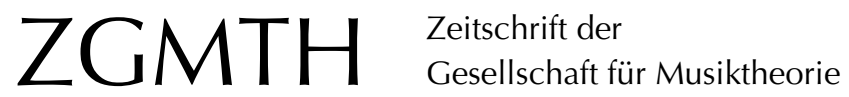

Schwab-Felisch, Oliver (2003/05): Zur Rezeption der Schichtenlehre Heinrich

Schenkers in der deutschsprachigen Musikwissenschaft nach 1945. ZGMTH 1-2/2/2-

3, 243-247. https://doi.org/10.31751/532

(C) 2003/05 Oliver Schwab-Felisch

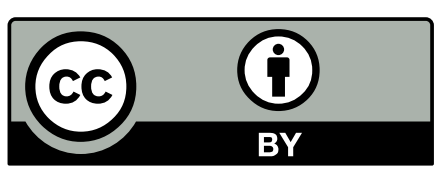

Dieser Text erscheint im Open Access und ist lizenziert unter einer Creative Commons Namensnennung 4.0 International Lizenz.

This is an open access article licensed under a

Creative Commons Attribution 4.0 International License.

veröffentlicht / first published: 01/04/2005

zuletzt geändert / last updated: 15/01/2010 


\title{
Zur Rezeption der Schichtenlehre Heinrich Schenkers in der deutschsprachigen Musik- wissenschaft nach 1945
}

\author{
Oliver Schwab-Felisch
}

Die Schichtenlehre Heinrich Schenkers ist im deutschsprachigen Raum bekanntlich deutlich zurückhaltender aufgenommen worden als in den angelsächsischen Ländern. Während das RILM etwa doppelt so viele englischsprachige wie deutschsprachige Publikationen zur Musiktheorie nachweist, liegt deren Verhältnis bei Schenker-Literatur bei rund 6:1.

Die Relevanz angelsächsischer Diskussionen für den deutschsprachigen Schenker-Diskurs ist gering. Zwar liegt die Zahl der in deutschsprachigen Zeitschriften erschienenen Verweise auf englischsprachige Schenker-Literatur mit insgesamt 83 Zitaten für die Zeit von 1975-2000 annähernd genauso hoch wie die Zahl der references auf deutschsprachige Schenker-Literatur - scheinbar das Zeichen einer gewissen Offenheit für den angelsächsischen Diskurs. Doch zeigt sich bei genauerem Hinsehen ein anderes Bild. Erstens ist die Gruppe der beteiligten Autoren wesentlich kleiner, als es die Zahlen vermuten lassen: So zeichnete etwa Hellmut Federhofer über viele Jahre hinweg für nahezu jede deutschsprachige Rezension von Schenker-Literatur verantwortlich. Zweitens stammt eine Reihe von Zitaten aus deutschsprachigen Beiträgen englischsprachiger Autoren. ${ }^{1}$ Drittens und letztens setzen sich die wenigsten Beiträge direkt mit der Theorie Schenkers auseinander oder enthalten gar Analysen nach Schenker. Es wundert daher nicht, daß, sofern man dem Arts and Humanities Citation Index glauben möchte, herausragende Forscher wie Carl Schachter, Charles Burkhart oder Allen Cadwallader zwischen 1975 und 2000 kein einziges Mal in einer deutschsprachigen Zeitschrift zitiert wurden.

Einige der bald nach 1945 unternommenen vereinzelten Versuche, Schenker wieder ins Gespräch zu bringen, sind von besonderem Interesse, weil sie Formen der Schenker-Rezeption repräsentieren, die der spätere musikwissenschaftliche Mainstream verworfen und ausgesondert hat. Sie bezeichnen gewissermaßen das unrealisierte Potential der Schenker-Rezeption in Deutschland, ein Potential, von dem aus ein Licht auch auf die spätere faktische Rezeption fällt. Zwei Beiträge können hier als exemplarisch herangezogen werden: Hans Wingerts »Über die `Urlinieく und ihren Schöpfer. Eine Würdigung und Heinrich Hartmanns »Heinrich Schenker und Karl Marx«.

1 Siehe etwa Hinton 1988, 1990. 
Hans Wingert gehört zum Typus des Theoretikers, der Schenkers Lehre im vollen Bewußtsein ihrer Einbettung in eine bestimmte /Weltanschauung ‘ favorisiert. Die Erfahrung des Weltkrieges ist hierfür ausschlaggebend. Für Wingert wird Schenkers Methode zu einer Art universellen Therapeutikums. Sie sorgt für das »Bewußtsein eines inneren Gleichgewichtes [...], eines Eingebettetseins in eine göttliche Ordnung, das uns vor seelischer Verarmung, vor Gehetztsein und vor einer Wiederholung wahnwitziger Katastrophen bewahren könnte ${ }^{2}$. Wingert favorisiert Schenker nicht trotz, sondern wegen seines Glaubens an die Invarianz naturgegebener musikalischer Gesetze. Er glaubt an die normative Kraft der Lehre Schenkers, eine Kraft im übrigen, die auch den Irrtum der Neuen Musik zu berichtigen im Stande sei. Hier wie auch in vielen anderen Punkten erweist sich Wingert als treuer Schenkerianer.

Das genaue Gegenteil von Wingerts Position beschreibt der 1952 - dem Erscheinungsjahr von Salzers Structural Hearing und dessen Rezension durch Milton Babbitt - in der Österreichischen Musikzeitschrift publizierte Aufsatz von Heinrich Hartmann (1952), „Heinrich Schenker und Karl Marx«. Hartmann betont, um Schenker gerecht zu werden, müsse man den »wissenschaftlichen Teil« seines Werkes vom »unwissenschaftlichen Teil« trennen. Es gelte, Schenkers »Renommierereien beiseite zu lassen«, »das trübe Schenker'sche Elixier [zu] filtirieren und allein jene Gesetze [zu] betrachten, die Schenker in der klassischen Musik erkannt hat « ${ }^{3}$. Dann zeige sich die Urlinie als »das Bezugssystem der klassischen Musik « ${ }^{4}$. Zugleich verwirft Hartmann den normativen Anspruch Schenkers und das aus diesem abgeleitete Geschichtsbild. Er kann dies, weil er, Karl Marx folgend, Urlinien-Musik als ein Gebilde der bürgerlichen Klasse begreift, deren ökonomisch bedingter »Auflösungsprozeß« im 19. Jahrhundert zwar das Ende der Urlinie, doch keineswegs das Ende aller Musik bewirkt habe. Hartmanns Konsequenz, das Postulat dreier Musiktheorien, ist bemerkenswert: „Die Schenkersche Theorie ist die klassische Musiktheorie. Die Theorie Riemanns [gilt] für die Musik Wagners, Bruckners, Hugo Wolfs, Regers usw. Die Theorie Schönbergs [... ist die Theorie] für die moderne Musik. ${ }^{5}$

Der Typus Wingert mit seiner speziellen Spielart des Konservativismus blieb in der Nachkriegsgeschichte der deutschen Schenker-Rezeption ebenso ein Einzelfall wie das marxistische Pathos der Wissenschaftlichkeit, mit dem Hartmann auftrat. Die universitäre Musikwissenschaft zeigte sich überwiegend skeptisch, befaßte sich allerdings primär mit frühen und mittleren musiktheoretischen Schriften Schenkers (Kolneder 1958, Keller 1966), während Der Freie Satz nach seiner vernichtenden Rezension durch Carl Dahlhaus (1959) im Großen und Ganzen unbeachtet blieb. Nicht ohne Ironie ist es, daß Harald Kaufmann (1965) und Rudolf Frisius (1978), Autoren also, die der Figur Schenker weitgehend gerecht zu werden vermochten, insbesondere mit Arbeiten zur Musik des 20. Jahrhunderts hervorgetreten sind. Geprägt wurde das Gesicht des deutschen Schenkerismus allerdings über lange Jahre hinweg durch Hellmut Federhofer, der mit konservativen musikalischen und kulturpolitischen Anschauungen an die Öffentlichkeit trat und

2 Wingert 1950, 244.

3 Hartmann 1950, 46.

4 Ebd., 48.

5 Ebd., 52. 
auch die polemische Auseinandersetzung nicht scheute ${ }^{6}$, zugleich aber als respektiertes Mitglied der akademischen Gemeinschaft der Schenkerianischen Perspektive eine klar vernehmbare Stimme verlieh.

Die Argumente deutscher Schenker-Kritiker unterscheiden sich grosso modo nicht grundsätzlich von denen ihrer amerikanischen Kollegen. Schenkers politische Tiraden werden ebenso erwähnt wie seine Ablehnung Neuer Musik, sein statisches Geschichtsbild oder sein dogmatischer Wahrheitsanspruch. ${ }^{7}$ Gleichfalls nicht spezifisch, aber doch von prägender Wirkung ist der Umstand, daß viele deutsche Musikforscher die Schichtenlehre als etwas betrachten, was den gewohnten Umgang mit Musik nicht bereichert, sondern einengt: Die strukturelle Perspektive, so sagt man, entziehe dem schönen Detail seinen musikalischen Ausdruckswert, das Primat der Stimmführung raube der Harmonik ihre Eigengesetzlichkeit, die geforderte Eindeutigkeit lasse es nicht zu, die Komponenten des Satzes in ihrer Wechselwirkung und potentiellen Ambiguität aufzufassen. Die Skepsis betrifft nicht nur konkrete Ergebnisse analytischer Entscheidungen, sondern auch den Anspruch der Theorie auf Vollständigkeit und Systematik. Darin aber hat die Kritik am Schenkerismus ihr allgemeines Moment. Nicht wenige deutschsprachige Musikwissenschaftler empfinden das Verhältnis zur Methode musikalischer Analyse als das wichtigste distinktive Merkmal englischsprachiger Musikforschung. Die methodisch strenge theoriebasierte Analyse, seit Milton Babbitt ein Leitbild nordamerikanischer Musiktheorie, wird in Deutschland vielfach nicht als Ausweis akademischer Respektabilität, sondern als einseitig und verkürzend aufgefaßt. Der Fokus musikalischer Analyse, so argumentiert man, liege in der Erkenntnis des individuellen Werkes. Eine Methode sei lediglich ein Instrument zu diesem Zweck. Sie müsse an einem bestimmten Punkt der Untersuchung allein schon deshalb fallengelassen werden, weil sich die Werke bereits auf struktureller Ebene aus verschiedenen historisch vermittelten Momenten zusammensetzten. Zudem bedürfe jede Methode eines äußeren Korrektivs. Denn die immanente Logik der Theorie neige dazu, sich gegenüber ihrem Gegenstand zu verselbständigen und damit Mißinterpretationen, Fehleinschätzungen und Verzerrungen hervorzubringen. ${ }^{8}$

Bei jüngeren Vertretern des Faches freilich nimmt die Bereitschaft zur Auseinandersetzung mit Schenker und der angelsächsischen Schenker-Diskussion erkennbar zu. Den Anfang machte Martin Eybls Dissertation zum »Verhältnis von Theorie und Ideologie in Schenkers Schichtenlehre« (1995). Mit dem an der Wiener Universität für Musik und darstellende Kunst beheimateten Lehrgang für Tonsatz nach Heinrich Schenker leitet Eybl das einzige der Erforschung und Vermittlung der Schichtenlehre gewidmete universitäre Institut im deutschsprachigen Raum. Zu nennen ist weiterhin Michael Polth (2000), dessen Dissertation ein an Schenker geschultes analytisches Denken in den Dienst gattungsgeschichtlicher Differenzierung stellt. Bernd Redmanns Dissertation zur Methodologie musikalischer Analyse (2002) wurde wesentlich durch Schenkers Schriften angeregt und enthält vielfältige Bezüge auf die Schichtenlehre. Redmann arbeitet gegenwärtig zur

6 Siehe etwa Federhofer 1981.

7 Siehe Dahlhaus 1959, 1962, 1984; Golab 1988.

8 Siehe hierzu auch Oliver Schwab-Felisch, »Analyse nach Heinrich Schenker», in: Systeme der Musiktheorie, hg. von Clemens Kühn, Dresden (in Vorbereitung). 
Rezeption Schenkers in Deutschland. Oliver Schwab-Felisch (2005) legte den Versuch einer Gesamtdarstellung des Forschungsfeldes vor, in der auch neuere Entwicklungen der angelsächsischen Schenkerian Analysis berücksichtigt werden. Patrick Boenke, ehemaliger Schüler Eybls und gegenwärtig Dozent am Wiener Schenker-Lehrgang, nutzt analytische Techniken Schenkers zur Untersuchung später Klavierwerke Liszts. ${ }^{9}$ Bernhard Haas und Veronica Diederen schließlich erarbeiten auf der Grundlage detaillierter Analysen Bachscher Inventionen ein System struktureller Schichten, in dem jede Schicht anhand bestimmter charakteristischer Erscheinungen absolut definiert werden kann. ${ }^{10}$

\section{Literatur}

Boenke, Patrick, „Klangprolongation und -projektion im späten Klavierwerk Franz Liszts«, in: Schenkerian Analysis - Analyse nach Heinrich Schenker. Bericht über den internationalen Schenker-Kongreß in Berlin, Sauen und Mannheim, 4.-12. Juni 2004, hg. von Oliver Schwab-Felisch, Michael Polth und Hartmut Fladt, 2 Bde., Hildesheim u. a.: Olms, erscheint 2007.

Dahlhaus, Carl (1959), Besprechung von Heinrich Schenker, Der Freie Satz, Die Musikforschung 12, 524.

— (1962), »Bach und der slineare Kontrapunkt«", Bach-Jahrbuch 49, 58-79.

(1978), "Schönberg und Schenker«, in Ders.: Schönberg und andere. Gesammelte Aufsätze [1965-1978] zur Neuen Musik, Mainz: Schott.

_ (1983), »Im Namen Schenkers«, Die Musikforschung 36, 82-87.

Diederen, Veronica / Bernhard Haas, Die zweistimmigen Inventionen von J. S. Bach, erscheint in Kürze.

Eybl, Martin(1995), IdeologieundMethode. Zumideengeschichtlichen KontextvonSchenkers Musiktheorie (Wiener Veröffentlichungen zur Musikwissenschaft 32), Tutzing: Schneider.

Federhofer, Hellmut (1950), Beiträge zur musikalischen Gestaltanalyse, Graz, Innsbruck und Wien: Akademische Druck- und Verlagsanstalt.

— (1981), Akkord und Stimmführung in den musiktheoretischen Systemen von H. Riemann, E. Kurth und H. Schenker, Wien: Akademie der Wissenschaften.

— (2004), »Heinrich Schenkers Vermächtnis«, Musiktheorie 19, 44-52.

Frisius, Rudolf (1978), „Vorwort« zu Heinrich Schenker, Harmonielehre. Neue musikalische Theorien und Phantasien I, Wien: Universal Edition.

Golab, Macicj (1988), »Analyse und Werk: Zu den Polemiken über Heinrich Schenkers Theorie», International Review of the Aesthetics and Sociology of Music 19, 197-215.

Grünzweig, Werner (1993), „Vom `Schenkerismus` zum >Dahlhaus-Projektı: Einflüsse deutschsprachiger Musiker und Musikwissenschaftler in den Vereinigten Staaten - Anfänge und Ausblick», Österreichische Musikzeitschrift 48, 161-170.

9 Boenke in Vorbereitung.

10 Haas / Diederen in Vorbereitung. 
Hartmann, Heinrich (1952), »Heinrich Schenker und Karl Marx«, Österreichische Musikzeitschrift 7, 46-52.

Hinton, Stephen (1988), »Musikwissenschaft und Musiktheorie oder: Die Frage nach der phänomenologischen Jungfräulichkeit«, Musiktheorie 2, 195-204.

— (1990), »`Natürliche Übergänge`. Heinrich Schenkers Begriff von der Sonatenform«, Musiktheorie 5, 101-116.

Kaufmann, Harald (1965), »Fortschritt und Reaktion in der Lehre Heinrich Schenkers«, Neue Zeitschrift für Musik 126, 5-9.

Keller, Wilhelm (1966), »Heinrich Schenkers Harmonielehre«, in: Beiträge zur Musiktheorie des 19. Jahrhunderts (Studien zur Musikgeschichte des 19. Jahrhunderts 4), hg. von Martin Vogel, Regensburg: Bosse, 203-232.

Köhler, Rafael (1996), »Linie und Urlinie. Zur Methodendiskussion in der energetischen Musiktheorie», in: Zur Geschichte der musikalischen Analyse, hg. von Gernot Gruber, Laaber: Laaber, 157-176.

Kolneder, Walter (1958), „Sind Schenkers Analysen Beiträge zur Bacherkenntnis?», Deutsches Jahrbuch für Musikwissenschaft 3, 59-73.

Polth, Michael (2000), „Sinfonieexpositionen des 18. Jahrhunderts. Formbildung und Ästhetik», Diss. Kassel: Bärenreiter.

— (2001), »Funktionalität?«, Musiktheorie 16, 319-324.

— (2002), »Individualität und Tonalität. Warum Schenkers Analysen die Individualität von Kompositionen zeigen«, in: Individualität in der Musik, hg. von Oliver SchwabFelisch, Christian Thorau und Michael Polth, Stuttgart: Metzler 2002, 69-93.

Redmann, Bernd (1996), „Zum (Schein-) Antipodentum von Hugo Riemann und Heinrich Schenker«, in: Zur Geschichte der musikalischen Analyse, hg. von Gernot Gruber, Laaber: Laaber, 131-144.

_ (2002), »Entwurf einer Theorie und Methodologie der Musikanalyse«, Diss. Laaber: Laaber.

Schwab-Felisch, Oliver (2005), »Zur Schichtenlehre Heinrich Schenkers», in: Handbuch der Systematischen Musikwissenschaft, Bd. 2: Musiktheorie, hg. von Helga de la Motte-Haber und Oliver Schwab-Felisch, Laaber: Laaber, 337-376.

Thaler, Lotte (1984), Organische Form in der Musiktheorie des 19. und beginnenden 20. Jahrhunderts (Berliner musikwissenschaftliche Arbeiten 25), München und Salzburg: Katzbichler.

Wingert, Hans (1950), „Über die „Urlinie` und ihren Schöpfer. Eine Würdigung«, Zeitschrift für Musik 111, 244-246.

Weitere Literaturhinweise in:

Berry, David Carson (2004), A Topical Guide to Schenkerian Literature. An Annotated Bibliography with Indices, Hillsdale, NY. Pendragon. 